\title{
Mechanism of Torque Ripple Generation by Time and Space Harmonic Magnetic Fields in Permanent Magnet Synchronous Motors
}

\author{
Katsumi Yamazaki, Member, IEEE, Kento Utsunomiya, and Hiroaki Ohiwa
}

Post Conference Paper

\begin{abstract}
In this article, we investigate mechanism of torque ripple generation by time and space harmonic magnetic fields in permanent magnet synchronous motors to obtain advanced motor designs. The general expression between the torque ripples and harmonic air-gap flux densities in the motor is derived by using Maxwell stress tensor. Both the numerical and experimental verifications of this expression are carried out. Then, the major harmonic magnetic fields that produce the torque ripples are specified and the differences between the surface and interior permanent magnet synchronous motors are investigated. According to these investigations, the shape of the rotor surface of an interior permanent magnet motor is optimized. It is clarified that specific harmonic components of the torque ripples in interior permanent magnet synchronous motors can be reduced to be nearly zero by optimizing the rotor surface shape.
\end{abstract}

Index Terms- Air gaps, optimization methods, permanent magnet motors, torque, finite element methods.

NOMENCLATURE

$B_{r}, B_{\theta}$ Radial and circumferential components of air-gap flux density

$B \quad$ A component of air-gap flux density $\left(B_{r}\right.$ or $\left.B_{\theta}\right)$

$\theta \quad$ Electric angle (stator coordinate system)

$t \quad$ Time

$\omega \quad$ Angular frequency

$k \quad$ Oder of space harmonics

$n \quad$ Order of time harmonics

$B_{k, n} \quad$ Amplitude of harmonic components included in $B$

$\varphi_{k, n} \quad$ Phase angle of harmonic components included in $B$

$\alpha_{\text {stator }}$ Modulation factor of air-gap flux density by stator harmonics

$\alpha_{j} \quad \mathrm{j}$-th harmonic component of $\alpha_{\text {stator }}$

$R \quad$ Radius of air gap surface between stator and rotor

$L \quad$ Core length

Manuscript received Month , 2021; revised Month xx, xxxx; accepted

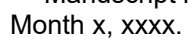

Katsumi Yamazaki, Kento Utsunomiya, and Horoaki Ohiwa are with the Department of Electrical and Electronic Engineering, Chiba Institute of Technology, Narashino, Chiba, 275-0016, Japan. (e-mail: yamazaki.katsumi@it-chiba.ac.jp).

\author{
$\mu_{0} \quad$ Vacuum permeability \\ $T \quad$ Total torque \\ $T_{m} \quad m$-th harmonic component included in waveform of $T$ \\ $W_{i} \quad$ Iron loss \\ $\sigma_{\max } \quad$ Maximum von Mises stress
}

\section{INTRODUCTION}

$\mathrm{M}$ AGNETIC field in permanent magnet synchronous motors (PMSMs) includes various time and space harmonics, which are generated by stator and rotor harmonic magnetomotive forces (MMFs). These harmonics produce harmonic losses and torque ripples. There are many articles that dealt with the reduction of these ill effects. The specific harmonic losses can be reduced by reducing the corresponding harmonic magnetic fields due to the requirement of each motor application [1], [2]. It can be stated that the mechanism of harmonic loss generation is straightforward and relatively simple. On the other hand, it is considered that the mechanism of torque ripple generation is more complex and still unclear.

Several articles reported the torque ripple reduction of PMSMs by control techniques based on circuit models with d-q axis decomposition [3], [4]. However, the cogging torque components, which are included in the torque ripples, cannot be represented by the circuit models because it generates without armature current. In reference [5], the magnetic co-energy term is added to the usual $\mathrm{d}-\mathrm{q}$ axis torque expression to take the effect of cogging torque into account. However, this term is not considered in the torque ripple minimization.

Also, there are many articles that report the reduction of cogging torques of PMSMs by improving the motor designs. For example, cogging torques of surface-mounted permanent magnet synchronous motors (SPMSMs) are often reduced by optimizing the shapes of permanent magnets (PMs) [6], [7]. This approach is very effective for SPMSMs, in which the harmonic magnetic field is directly determined by the surface shapes of the PMs. On the other hand, it is considered that the generation mechanism of cogging torques in interior permanent magnet synchronous motors (IPMSMs), which are widely used for variable speed/load applications, is more complex because the PMs are buried in the rotor core and the harmonic magnetic 
fields are considerably affected by the magnetic saturation. Furthermore, the saturated area in the rotor core considerably varies with the armature current. Therefore, the harmonic components of torque ripples under load conditions often become considerably different from those of cogging torques under no-load condition. Consequently, in many articles, the reduction of torque ripples of IPMSMs is often carried out by trial-and-error process of stator and/or rotor designs.

The total torque including the torque ripples of PMSMs can also be expressed by air-gap flux density due to Maxwell stress tensor [8]-[10]. However, there is no article that reports the general relationship between the torque ripples and the harmonic magnetic fields generated by the stator and the rotor. In references [11] and [12], the magnetic fields in motors are decomposed into time and space harmonics. However, the torque ripples have not been derived from these harmonic components. We consider that the generation mechanism of cogging torques and torque ripples in PMSMs can be clarified and the observations for advanced motor designs can be obtained, if they are directly expressed by the harmonic magnetic fields.

From these viewpoints, we have derived the general expression between the harmonic air-gap flux densities and torque ripples in [13] by using Maxwell stress tensor, to specify the harmonic fields that produce the torque ripples in IPMSMs, and to obtain advanced motor designs. In this article, this expression is applied to both the SPMSM and the IPMSM to clarify the difference in the generation mechanism of torque ripples. In addition, both the numerical and experimental verifications of the proposed expression are carried out. According to these investigations, the rotor surface shape of an IPMSM is optimized to reduce the specific components of torque ripples to be zero.

\section{HARMONIC FIELDS AND TORQUE RIPPLE EXPRESSION}

\section{A. Harmonic Magnetic Fields in PMSMs}

The air-gap flux density $B$ in rotating machines can be decomposed into time and space harmonic components by using Fourier transformation, as follows [12] :

$$
B=\sum_{k}^{\infty} \sum_{n}^{\infty} B_{k, n} \cos \left(k \theta-n \omega t+\varphi_{k, n}\right)
$$

where $k$ and $n$ are the space and time harmonic orders, respectively; $B_{k, n}$ and $\varphi_{k, n}$ are the amplitude and phase angles of the harmonic component, respectively; $\theta$ is the electric angle fixed to stator coordinates system, and $t$ is the time. Forward harmonic rotational fields are expressed by positive $n$, whereas the backward fields are expressed by negative $n$. Both the radial and circumferential components can be expressed by (1) with different amplitudes and phase angles.

Then, let us discuss the major harmonic magnetic fields existing in PMSMs. Fig. 1 shows a standing wave produced by the stator and a traveling wave synchronized with the rotor. The traveling wave consists of fundamental rotational field and harmonic components produced by harmonic rotor MMFs. The sum of these components $B_{\text {synchronized }}$ can be expressed, as follows:

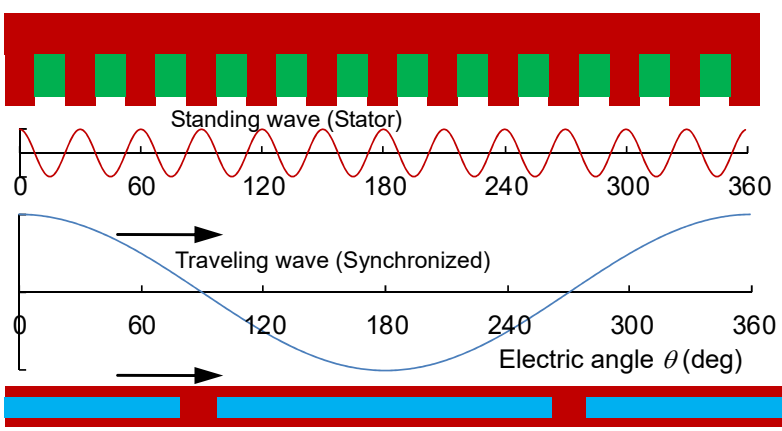

Fig. 1. Standing and traveling waves in PMSMs.

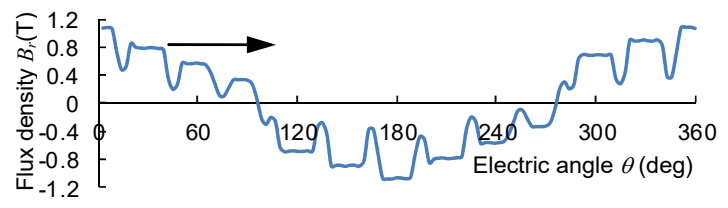

Fig. 2. Example of air gap flux density in PMSMs.

$$
B_{\text {Synchronized }}=\sum_{i}^{\infty} B_{i, i} \cos \left(i \theta-i \omega t+\varphi_{i, i}\right)
$$

The space harmonic orders $k$ of these components is equal to the time harmonic orders $n$ because they are synchronized. Therefore, both $k$ and $n$ are expressed by $i(=1,3,5 \ldots)$ in (2). The harmonic rotational fields caused by magnetic saturation are also included in (2).

On the other hand, the standing wave consists of phase-band harmonics and slot harmonics. It can be considered that the effects of these harmonics on the total air-gap flux density $B$ do not vary with $t$, whereas they vary with $\theta$ due to the space harmonic order. Therefore, we assume following factor, which expresses the total effect of the stator harmonics on $B$ :

$$
\alpha_{\text {stator }}=\sum_{j}^{\infty} \alpha_{j} \cos \left(j \theta+\varphi_{j, 0}\right)
$$

where $j$ is the space harmonic order, which is determined by the numbers of phases and stator slots. For example, $j=6,12,18 \ldots$, when the number of stator slots per pole pair is multiple of 6 and the number of phases is $3 . \alpha_{j}$ is the amplitude of $j$-th harmonic components included in $\alpha_{\text {stator }}$. Note that $\alpha_{\text {stator }}$ includes both the effects of slot permeance distribution and winding configuration.

Fig. 2 shows an example of air-gap flux density waveform in PMSMs. This waveform can be expressed by $B_{\text {synchronized }}$ modulated by $\alpha_{\text {stator }}$. Therefore,

$$
B=\sum_{i}^{\infty} B_{i, i}\left\{1+\sum_{j}^{\infty} \alpha_{j} \cos \left(j \theta+\varphi_{j, 0}\right)\right\} \cos \left(i \theta-i \omega t+\varphi_{i, i}\right)
$$

This expression can be expanded by mathematical formula of trigonometric functions, as follows:

$$
\begin{aligned}
& B=\sum_{i}^{\infty} B_{i, i} \cos \left(i \theta-i \omega t+\varphi_{i, i}\right) \\
& +\sum_{j}^{\infty} \sum_{i}^{\infty} \frac{1}{2} B_{i, i} \alpha_{j} \cos \left\{(j-i) \theta+i \omega t+\left(\varphi_{j, 0}-\varphi_{i, i}\right)\right\} \\
& +\sum_{j}^{\infty} \sum_{i}^{\infty} \frac{1}{2} B_{i, i} \alpha_{j} \cos \left\{(j+i) \theta-i \omega t+\left(\varphi_{i, i}+\varphi_{j, 0}\right)\right\}
\end{aligned}
$$


The first term is equal to (2). By comparing (1) and (5), it can be considered that the air-gap flux density of PMSMs includes following harmonic components:

a) Fundamental and harmonic rotational fields, which are synchronized with the rotor. They are expressed by the first term in (5) $(k=n=+i)$

b) The components generated by the modulation of above rotational fields by the stator standing wave. They are expressed by the second and third terms in (5).

( $k=j-i$ and $n=-i$ for the second term)

$(k=j+i$ and $n=+i$ for the third term)

Fig. 3 shows an example of predicted time and space harmonic orders included in the air-gap flux density of PMSMs, whose number of stator slots per pole pair is multiple of 6 and number of phases is 3 . As the space harmonic order $k$ is always defined to be positive, the signs of both $k$ and $n$ are reversed when $j-i$ is negative. This figure will be confirmed in section III.

Note that additional harmonic fields will be superimposed on $B$ when the motor is driven by inverters. The space harmonic orders of these harmonics are 1 and the time harmonic orders are around multiples of carrier frequency/fundamental frequency. For simplicity, these harmonic fields are not treated in this article.

\section{B. General Expression of Torque Ripples by Harmonics}

Next, the general expression of torque ripples by the harmonic magnetic fields is derived due to the discussion of section II-A and Maxwell stress tensor.

On the basis of 2D approximation, the total torque $T$ can be expressed by Maxwell stress tensor on the air-gap surface, as follows:

$$
T=R L \int_{\text {Gap }} \frac{1}{\mu_{0}}\left\{\left(u_{x}{ }^{2}-u_{y}{ }^{2}\right) B_{x} B_{y}+u_{x} u_{y}\left(B_{y}{ }^{2}-B_{x}{ }^{2}\right)\right\} d l
$$

where $R$ is the radius of air-gap surface that can be arbitrary selected between the stator and rotor surface, $L$ is the core length, $\mu_{0}$ is the vacuum permeability, $\left(u_{x}, u_{y}\right)$ and $\left(B_{x}, B_{y}\right)$ are the unit normal vector and the flux density vector on the air-gap surface, respectively. By transforming the rectangular coordinate system to the polar system, the expression (6) can be rewritten, as follows:

$$
T=\frac{R^{2} L}{\mu_{0}} \int_{0}^{2 \pi} B_{r} B_{\theta} d \theta
$$

where $B_{r}$ and $B_{\theta}$ are the radial and circumferential components of the air-gap flux density, respectively.

By applying Fourier transformation to $B_{r}$ and $B_{\theta}$ as (1), respectively, the expression (7) becomes

$$
\begin{array}{r}
T=\frac{R^{2} L}{\mu_{0}} \int_{0}^{2 \pi}\left\{\sum_{k_{r}}^{\infty} \sum_{n_{r}}^{\infty} B_{r, k_{r}, n_{r}} \cos \left(k_{r} \theta-n_{r} \omega t+\varphi_{r, k_{r}, n_{r}}\right)\right\} \\
\left\{\sum_{k_{\theta}}^{\infty} \sum_{n_{\theta}}^{\infty} B_{\theta, k_{\theta}, n_{\theta}} \cos \left(k_{\theta} \theta-n_{\theta} \omega t+\varphi_{\theta, k_{\theta}, n_{\theta}}\right)\right\} d \theta
\end{array}
$$

where $k_{r}, k_{\theta}, n_{r}$, and $n_{\theta}$ are the space and time harmonic orders of $B_{r}$ and $B_{\theta}$, respectively; $B_{r, k r, n r}, B_{\theta, k \theta, n \theta,} \varphi_{r, k r, n r}$ and $\varphi_{\theta, k \theta, n \theta}$ are the amplitudes and phase angles of the harmonic $B_{r}$ and $B_{\theta}$,

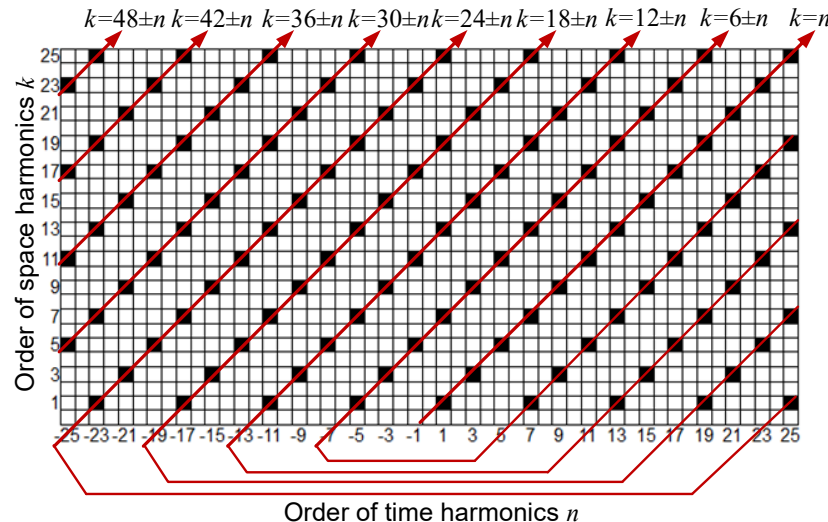

Fig. 3. Predicted time and space harmonic orders included in air gap flux density.

respectively. Due to the orthogonality of trigonometric functions, the integral of the cosine products by $\theta$ becomes zero when $k_{r}$ is not equal to $k_{\theta}$. Therefore, the expression (8) can be written, as follows:

$$
\begin{gathered}
T=\frac{R^{2} L}{\mu_{0}} \sum_{k}^{\infty} \int_{0}^{2 \pi}\left\{\sum_{n_{r}}^{\infty} B_{r, k, n_{r}} \cos \left(k \theta-n_{r} \omega t+\varphi_{r, k, n_{r}}\right)\right\} \\
\left\{\sum_{n_{\theta}}^{\infty} B_{\theta, k, n_{\theta}} \cos \left(k \theta-n_{\theta} \omega t+\varphi_{\theta, k, n_{\theta}}\right)\right\} d \theta
\end{gathered}
$$

After applying the cosine product-to-sum formula to (9), and carrying out the integral by $\theta$, the final expression is obtained, as follows:

$$
T=\frac{\pi R^{2} L}{\mu_{0}} \sum_{k}^{\infty} \sum_{n_{r}}^{\infty} \sum_{n_{\theta}}^{\infty} B_{r, k, n_{r}} B_{\theta, k, n_{\theta}} \cos \left\{\left(n_{\theta}-n_{r}\right) \omega t+\varphi_{r, k, n_{r}}-\varphi_{\theta, k, n_{\theta}}\right\}
$$

This is the general expression between torque and harmonic air-gap flux densities. For example, it implies that the $m$-th harmonic component included in the torque waveform ( $m$-th torque ripple) is generated by the product of the harmonic components of $B_{r}$ and $B_{\theta}$, whose space harmonic orders are identical to each other and the difference in the time harmonic orders $\left(n_{\theta}-n_{r}\right)$ is equal to $\pm m$.

\section{APPLICATION OF THEORY TO PMSMS}

\section{A. Numerical and Experimental Verifications}

First, numerical and experimental verifications of the torque expression described in section II are carried out by applying the theory to a SPMSM, in which the mechanism of torque ripple generation is considered to be relatively simple. 2D nonlinear time-stepping finite element analysis (FEA) is applied to the calculation of harmonic fields and torques.

Table I lists the motor specification. Fig. 4 shows the cross section. The motor has one sintered Sm-Co magnet per pole. The numbers of phases and stator slots per pole pair are 3 and 6, respectively. This motor was manufactured by IEE-Japan rotating machine committee for the purpose of confirming the accuracy of measured and calculated cogging torques.

Fig. 5 shows the experimental and calculated cogging torque waveform (waveform of $T$ at no load without armature current). The figure indicates not only the calculated result by 
TABLE I

MOTOR SPECIFICATION (SPMSM).

\begin{tabular}{c|c}
\hline Phases and poles & 3 phases, 4 poles \\
\hline Rated rotational speed & $1500 \mathrm{r} / \mathrm{min}$ \\
\hline Diameter of stator, Core length & $26.7 \mathrm{~mm}, 37.5 \mathrm{~mm}$ \\
\hline Number of stator slot & $12($ concentrated $)$ \\
\hline Magnet type, Magnetization & Sm-Co, $0.9 \mathrm{~T}$ \\
\hline Thickness of electrical steel sheet & $0.5 \mathrm{~mm}$ \\
\hline
\end{tabular}

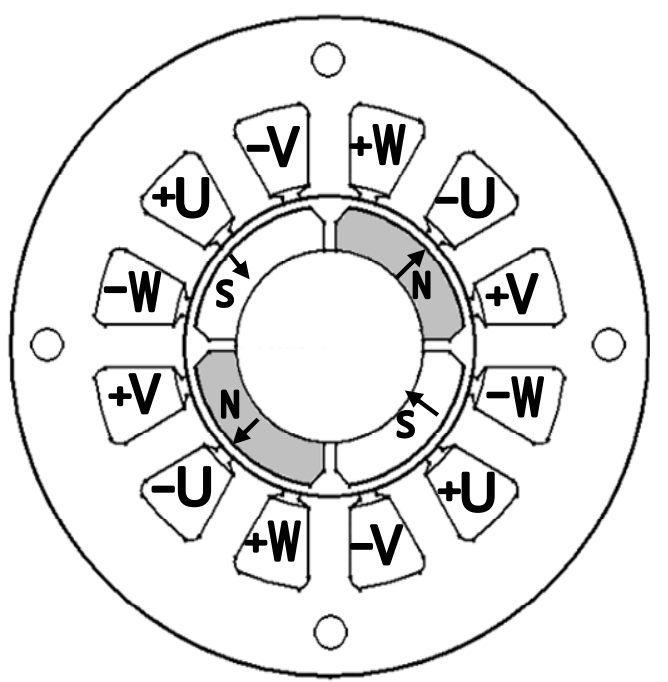

Fig. 4. Cross section of SPMSM.

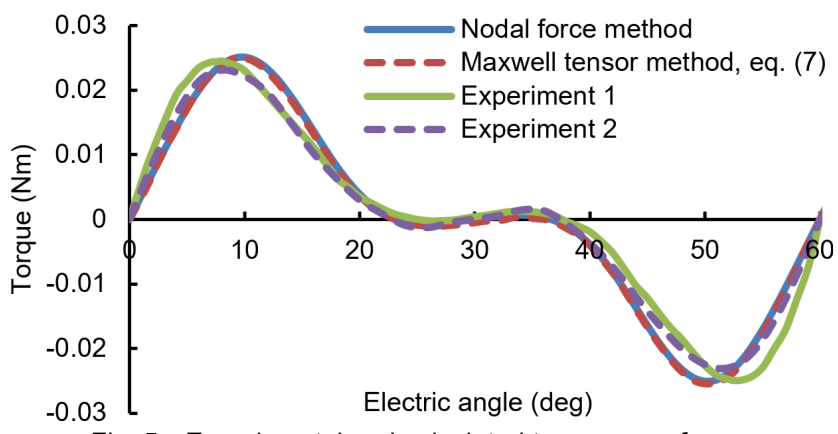

Fig. 5. Experimental and calculated torque waveforms (SPMSM, no load).

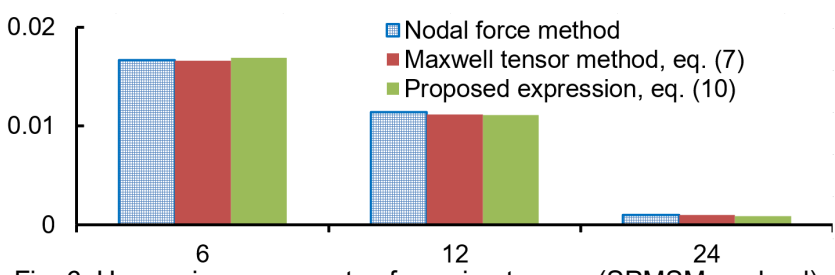

Fig. 6. Harmonic components of cogging torque. (SPMSM, no load).

the Maxwell tensor method by (7), but also that by the nodal force method, which is often used in commercial software. In the nodal force method, the torque is calculated by the summation of the force acted on the nodes of the finite elements in the rotor region [14]. These calculated results are nearly identical to each other. The figure also indicates the experimental results by two different laboratories. It is confirmed that the calculated results are found to be in good agreement with these experimental results.

Fig. 6 shows the amplitudes of the cogging torque $T_{6}, T_{12}$, and $T_{24}$, which are the 6,12 , and $24^{\text {th }}$ components extracted from the torque waveforms in Fig. 5. The results by (10) are
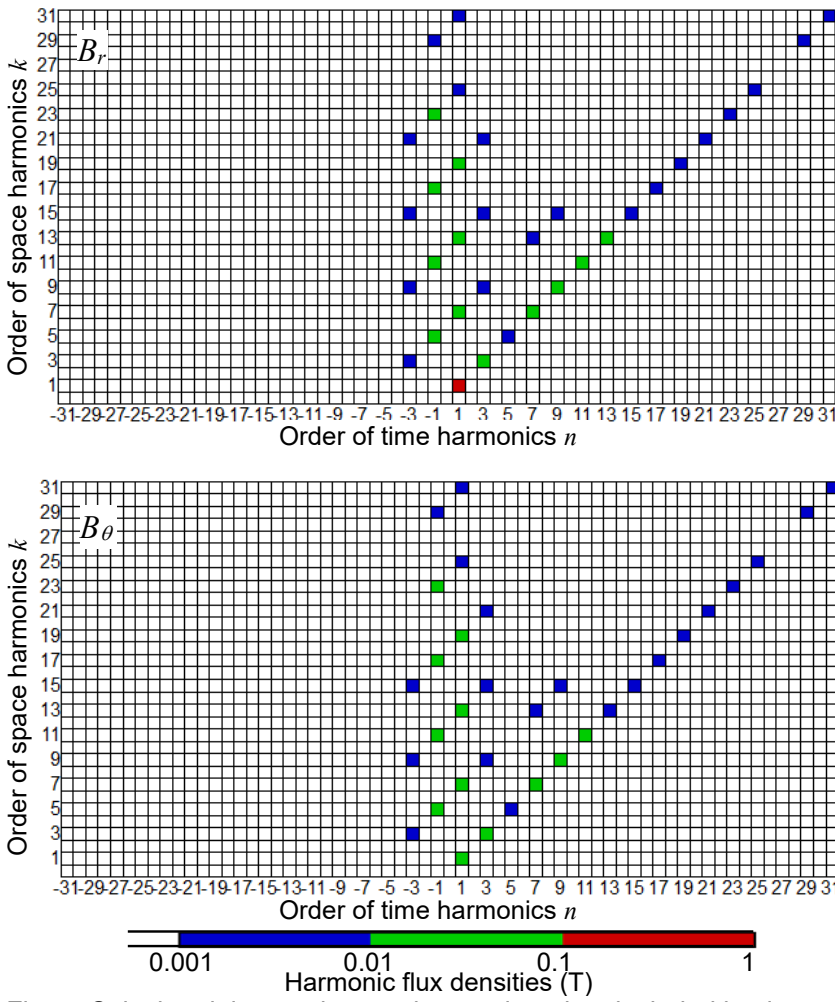

Fig. 7. Calculated time and space harmonic orders included in air gap flux density (SPMSM, no load).

also shown. In this expression, the torque ripples are obtained by the sum of the harmonic products whose $\left(n_{\theta}-n_{r}\right)$ is 6,12 , and 24 , respectively. The results by (10) are also found to be in good agreement with those by (7) and the nodal force method.

Fig. 7 shows the time and space harmonic components included in $B_{r}$ and $B_{\theta}$ of the air-gap flux density in this case. These components can be calculated by using the method described in [12]. As it was predicted in Fig. 3, the space harmonic orders of the major harmonics are expresses as $k=i$ $(i=1,3,5 \ldots$, synchronized harmonic rotational magnetic fields) and $k=j \pm i(j=6,12,18 \ldots$, the components generated by the modulation of $i$-th harmonic rotational field by $j$-th stator harmonic fields). Due to (10), the $m$-th cogging torque is generated by the sum of all the combination of harmonic $B_{r}$ and $B$, whose space harmonic orders are identical to each other and the difference in the time harmonic orders $\left(n_{\theta}-n_{r}\right)$ is equal to $\pm m$. From these results, the theory described in section II is verified.

Fig. 8 shows the distributions of $B_{r}$ and $B_{\theta}$ at $t=0$. The included space harmonics are also shown. It is observed that all the harmonic components of $B_{r}$ are \pm sine curves, whereas those of $B_{\theta}$ are \pm cosine at $t=0$. These facts implies that the phase angle $\varphi_{r, k r, n r}$ in (8)-(10) under the no-load condition is 90 or 270 $\mathrm{deg}$, whereas $\varphi_{\theta, k \theta, n} \theta$ is 0 or $180 \mathrm{deg}$, for any harmonic orders.

\section{B. Components of Cogging Torque and Torque Ripples}

Then, let us discuss the major harmonic fields that produce the cogging torques and torque ripples. The harmonic fields of an IPMSM are also investigated and the results are compared with those of the SPMSM described in section III-A.

Table II lists the specification of the IPMSM. Fig. 9 shows 


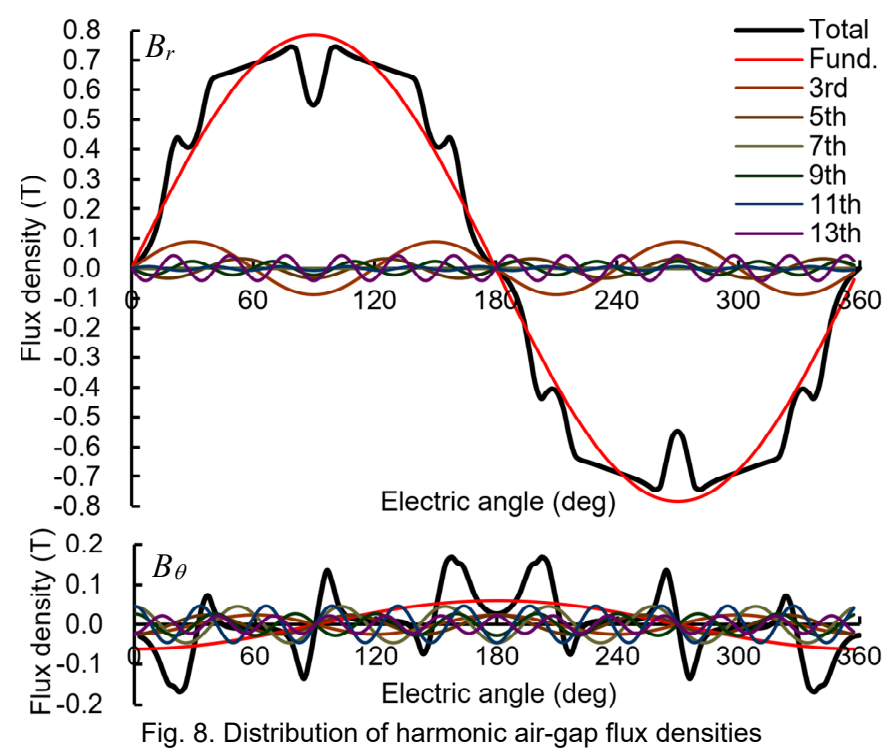

Fig. 8. Distribution of harmonic air-gap flux densities (SPMSM, no load, $t=0$ )

the cross section. The motor has 3 sintered Nd-Fe-B magnets per pole. The number of stator slots per pole pair is 12 . This motor is designed for variable speed/load applications.

Fig. 10 shows the variation in torque waveforms of the SPMSM and the IPMSM with load. The armature current of SPMSM under the load condition is set to be $3 \mathrm{~A}$, whereas that of the IPMSM is $88 \mathrm{~A}$, which is one of the typical condition of this motor. It is observed that both the phase angle and the amplitude of the torque ripples under the load condition varies from those of the cogging torques under the no-load condition, particularly in the case of the IPMSM.

Fig. 11 shows the phasor diagrams of the components that produce $T_{6}, T_{12}$, and $T_{24}$ decomposed by the proposed expression (10). In this figure, the components in (10) are expressed by the blue circles, whose distance from the origin corresponds to the amplitude, whereas the angle corresponds to the phase angle of each component. The sum of these components is also shown by the red squares, whose distance from the origin is equal to the result of Fig. 6 in the case of the SPMSM under the no load condition. It is clarified that the cogging torques and torque ripples are generated by considerable number of the combinations of harmonic $B_{r}$ and $B_{\theta}$ in both the SPMSM and the IPMSM. There are 4 major components in each order $T_{m}$, as follows:

$$
\begin{aligned}
T_{m} \cong & \frac{\pi R^{2} L}{\mu_{0}} B_{r, m \pm 1, m \pm 1_{r}} B_{\theta, m \pm 1, \pm 1} \cos \left(m \omega t+\varphi_{r, m \pm 1, m \pm 1}-\varphi_{\theta, m \pm 1, \pm 1}\right) \\
& +\frac{\pi R^{2} L}{\mu_{0}} B_{r, m \pm 1, \pm 1_{r}} B_{\theta, m \pm 1, m \pm 1} \cos \left(m \omega t+\varphi_{r, m \pm 1, \pm 1}-\varphi_{\theta, m \pm 1, m \pm 1}\right)
\end{aligned}
$$

As discussed in section II-A, $\left(B_{r, m \pm 1, m \pm 1}, B_{\theta, m \pm 1, m \pm 1}\right)$ is produced by rotor harmonics, which are determined by the rotor shape and permeability. On the other hand, $\left(B_{r, m \pm 1, \pm 1}, B_{\theta, m \pm 1, \pm 1}\right)$ is produced by the modulation of fundamental rotational field $B_{1,1}$ by the $m$-th stator standing wave, which is phase-band harmonics or slot harmonics. It is observed that $T_{6}$ of the IPMSM under the no-load condition is zero, whereas that under the load condition is not negligible. The reason is that all the components of $T_{6}$ in the IPMSM are generated only by the phase-band harmonics, which are produced by three phase currents, because the number of stator slots per pole pair is 12 .
TABLE II

MOTOR SPECIFICATION (IPMSM).

\begin{tabular}{c|c}
\hline Phases and poles & 3 phases, 8 poles \\
\hline Maximum rotational speed & $10000 \mathrm{r} / \mathrm{min}$ \\
\hline Diameter of stator, Core length & $200 \mathrm{~mm}, 150 \mathrm{~mm}$ \\
\hline Number of stator slots & $48($ distributed $)$ \\
\hline Magnet type, Magnetization & Nd-Fe-B, $1.1 \mathrm{~T}$ \\
\hline Thickness of electrical steel sheet & $0.3 \mathrm{~mm}$ \\
\hline
\end{tabular}

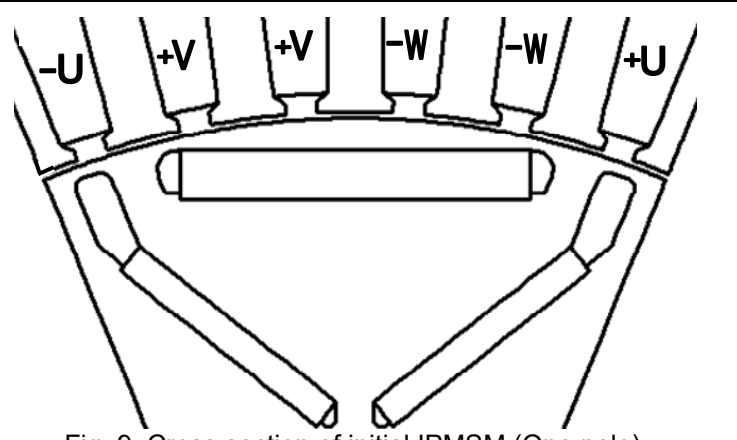

Fig. 9. Cross section of initial IPMSM (One pole).

Fig. 11 also indicates that the phase angles of all the torque components under the no-load condition are 90 or $270 \mathrm{deg}$. The reason is that these phase angles are expressed in (10) as the difference between the phase angles of harmonic $B_{r}$ and $B_{\theta}$ $\varphi_{r, k r, n r}-\varphi_{\theta, k \theta, n}$. As shown in Fig. 8, $\varphi_{r, k r, n r}$ under the no-load condition is 90 or $270 \mathrm{deg}$, whereas $\varphi_{\theta, k \theta, n \theta}$ is 0 or $180 \mathrm{deg}$. Therefore, $\varphi_{r, k r, n r}-\varphi_{\theta, k \theta, n \theta}$ becomes 90 or $270 \mathrm{deg}$. On the other hand, these phase angles considerably vary with load. This variation is caused by the variation in $\varphi_{r, k r, n r}$ and/or $\varphi_{\theta, k \theta, n \theta}$.

To understand the detailed mechanism of this variation, the variation in the harmonic magnetic fields with load is investigated. Fig. 12 shows the phasor diagram of harmonic fields that cause two major components of $T_{12}\left(B_{r, 11,11} B_{\theta, 11,-1}\right.$ and $\left.B_{r, 13,13} B_{\theta, 13,1}\right)$. In the SPMSM, $B_{r, 11,11}$ and $B_{r, 13,13}$, which are produced by harmonic rotor MMF, are almost constant with load. Only the phase angles of $B_{\theta, 11,-1}$ and $B_{\theta, 13,1}$, which are produced by the modulation of fundamental $B_{1,1}$ by the stator standing wave, are changed. This variation is mainly caused by the variation in $B_{1,1}$ by the armature reaction under the load condition. On the other hand, in the case of the IPMSM, not only $B_{\theta, 11,-1}$ and $B_{\theta, 13,1}$, but also $B_{r, 11,11}$ and $B_{r, 13,13}$ vary with load. This variation must have been caused by the variation in the rotor permeability distribution with load. Fig. 13 shows the calculated relative permeability distribution of the IPMSM. It is observed that the distribution considerably varies with load, particularly at the rotor core surface. As a result, the rotor harmonics varied. On the other hand, in the SPMSM, it is considered that the rotor harmonics are not considerably changed with load because there is no core at the rotor surface. It can be stated that the mechanism of the torque ripple generation in IPMSMs is more complex than SPMSMs because of the variation in all the harmonic magnetic fields with load.

\section{TORque Ripple Reduction BY MOtOR OptimizATION}

\section{A. Strategy of Torque Ripple Reduction}

Finally, the reduction of the torque ripples in the IPMSM is investigated by optimizing the motor shape. From the observations obtained in section III, three strategies can be considered to reduce the $m$-th torque ripples of the IPMSM, as follows: 

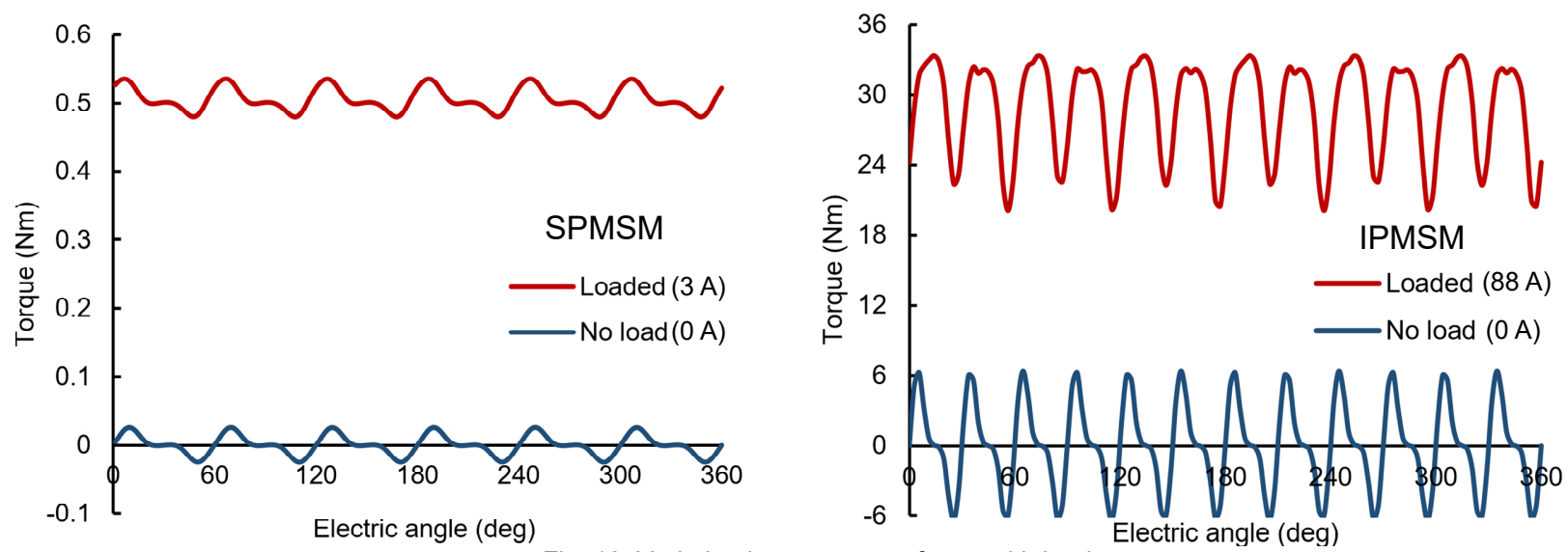

Fig. 10. Variation in torque waveforms with load.
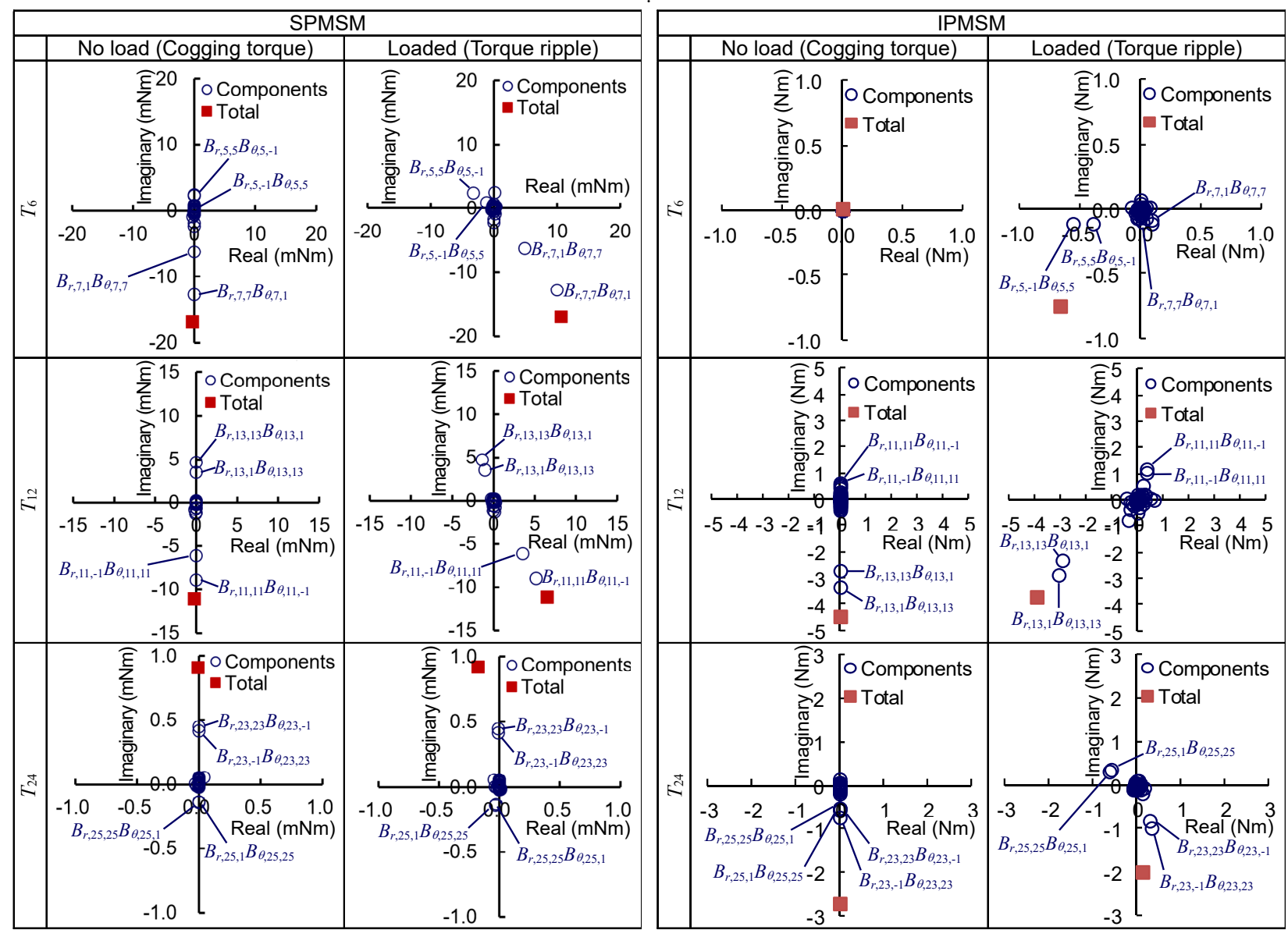

Fig. 11. Components of cogging torques and torque ripples.
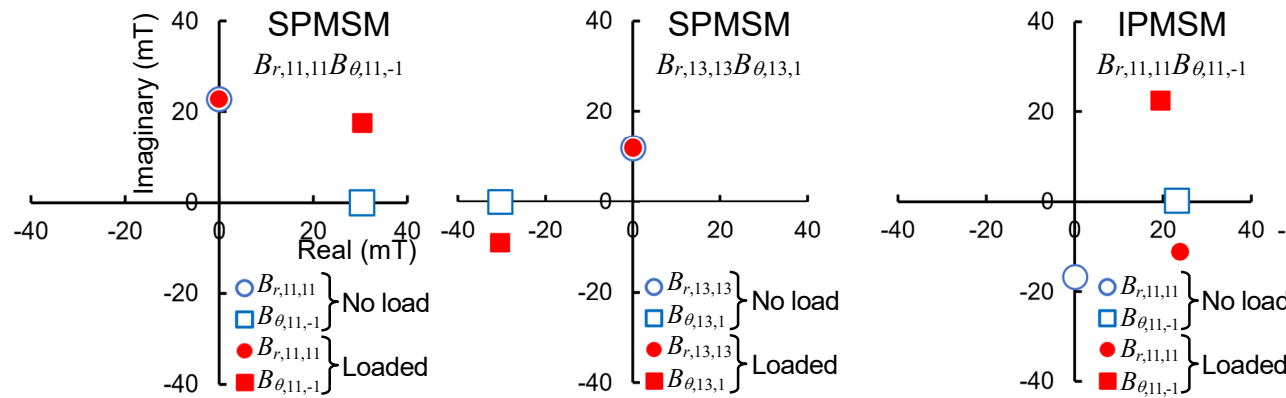

Fig. 12. Harmonic fields that cause major torque ripple components

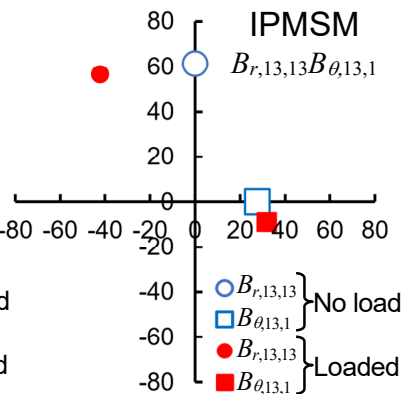




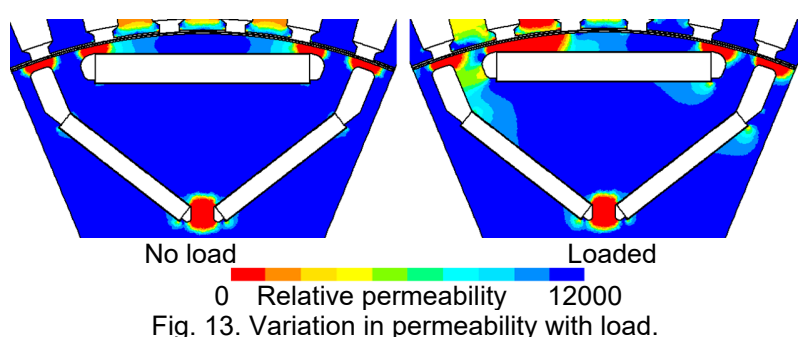

a) Decrease the rotor harmonics $\left(B_{r, m \pm 1, m \pm 1}, B_{\theta, m \pm 1, m \pm 1}\right)$ by optimizing rotor shape.

b) Decrease $m$-th standing wave produced by the stator.

c) Decrease the sum of components in the $m$-th torque ripple in

Fig. 11 by optimizing rotor shape and/or stator-slot shape.

In this article, the strategy (c) with the modification of rotor surface shape is adopted because the rotor harmonics in IPMSMs significantly vary with the air gap. It is expected that the torque ripple can be reduced by slight modification of the rotor surface shape without considerable deteriorations of the other important motor characteristics.

\section{B. Conditions of Automatic Optimization}

Then, the automatic rotor surface optimizations are carried out to decrease the torque ripples under frequent driving conditions in the variable speed/load application.

Fig. 14 shows the rotor surface points, whose radial and circumferential coordinates are selected as the design variables. The circumferential coordinates of $\mathrm{P}_{1}$ and $\mathrm{P}_{11}$ are fixed to be 0 and $22.5 \mathrm{deg}$, respectively to keep the rotor shape symmetric.

Table III lists the estimated characteristics and driving conditions. The condition A is a typical low speed and low torque condition (the same condition in Figs. 10 to 13), whereas the condition $\mathrm{B}$ is the instantaneous maximum torque condition. The average torque $\left(T_{0}\right)$, and the torque ripples $\left(T_{6}\right.$, $\left.T_{12}, T_{24}\right)$ are estimated under these conditions. The torque ripple reduction is particularly required under the condition A because the driving term of the analyzed motor around this condition is very long. On the other hand, the condition $\mathrm{C}$ is the maximum speed condition under flux weakening control with rated armature current. The iron loss $W_{i}$ and the maximum von Mises stress $\sigma_{\max }$ by centrifugal force are estimated under this condition because they considerably increase with the rotational speed.

Fig. 15 shows the calculation system for the automatic shape optimization [15]. Both the 2D stress FEA (under the condition $\mathrm{A}$ ) and the 2D electromagnetic field FEA (under the condition A, B, and C) are carried out due to the rotor shape determined by Rosenbrock's method. Then, the torques, iron loss, and maximum von Mises stress are fed back to Rosenbrock's method as the objective function or constraint conditions in order to determine the optimized shape.

\section{C.Results of Optimizations}

First, the object function is set to be the total torque ripple under condition $\mathrm{A}$, which is defined by the difference between the maximum and minimum torques in the torque waveform. In this case, the constraint conditions are imposed, as follows:

a) $T_{0}$ under both the conditions $\mathrm{A}$ and $\mathrm{B}$ should be larger than $97 \%$ of those of the initial design, respectively.

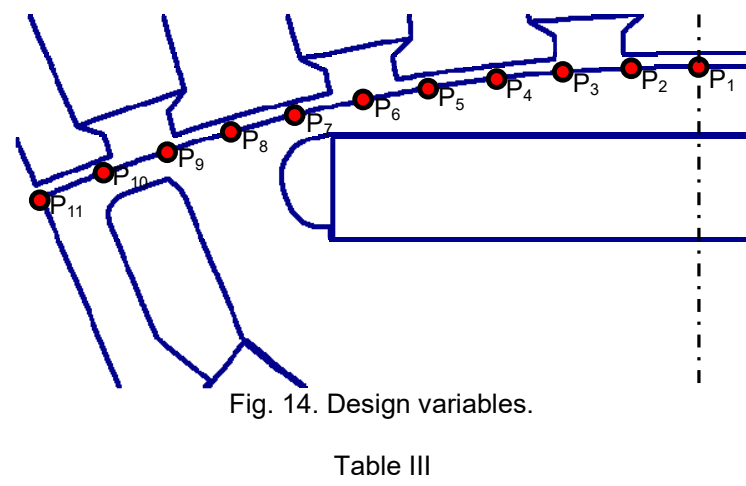

Estimated characteristics and driving conditions.

\begin{tabular}{c|c|c|c}
\hline & Condition A & Condition B & Condition C \\
\hline Rotational speed (r/min) & 2500 & 2500 & 10000 \\
\hline Armature current (A) & 88 & 700 & 300 \\
\hline Estimated characteristics & $T_{0}, T_{6}, T_{12}, T_{24}$ & $T_{0}, T_{6}, T_{12}, T_{24}$ & $W_{i}, \sigma_{\max }$ \\
\hline
\end{tabular}

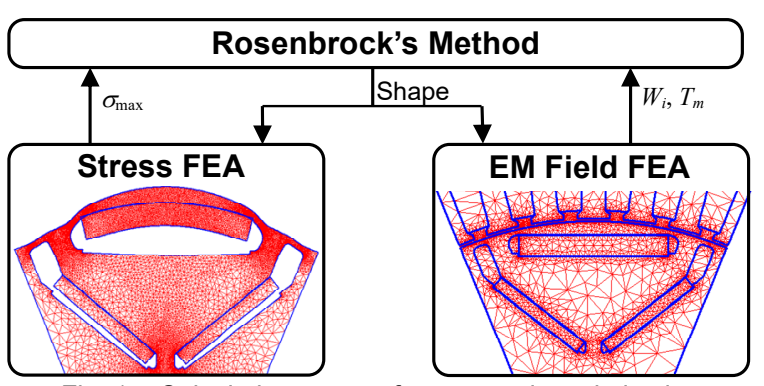

Fig. 15. Calculation system for automatic optimization.
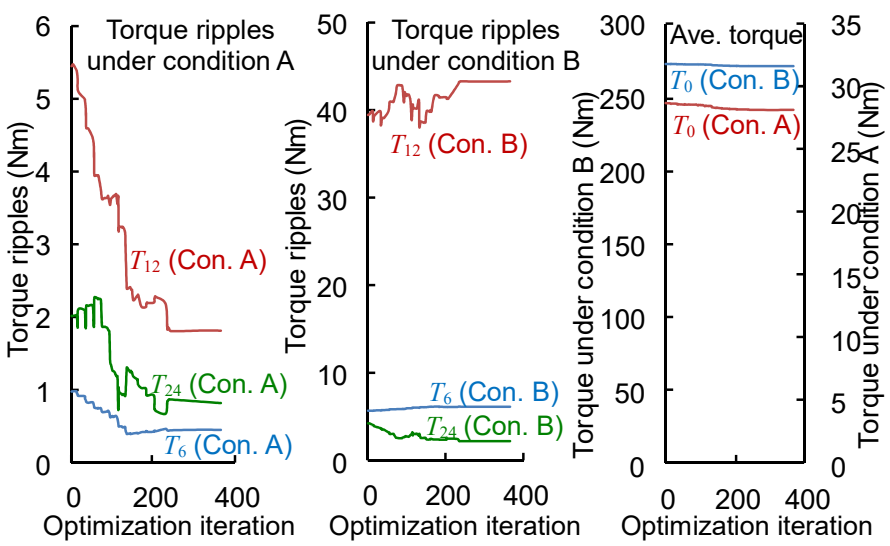

Optimization iteration Optimization iteration Optimization iteration
Fig. 16. Minimization of total torque ripple under driving condition A.

b) $T_{6}, T_{12}, T_{24}$ under the condition B should be less than $130 \%$ of those of the initial design, respectively.

c) $W_{i}$ under the condition $\mathrm{C}$ should be less than that of the initial design.

d) $\sigma_{\max }$ under the condition $\mathrm{C}$ should be less than $300 \mathrm{MPa}$.

Fig 16 shows the variation in $T_{6}, T_{12}, T_{24}$, and $T_{0}$ with optimization iterations. It is observed that all the torque ripples under the condition A are reduced by this optimization, whereas the other characteristics satisfy the constraint conditions. In this case, the total torque ripple is reduced to be nearly $1 / 3$ of that of the initial design.

Next, three additional optimizations, whose objective functions are $T_{6} T_{12}$ and $T_{24}$ under the condition $\mathrm{A}$, are individually carried out. It is because the reduction of specific order torque ripple is often strongly desired due to the requirement of the mechanical system in each motor 

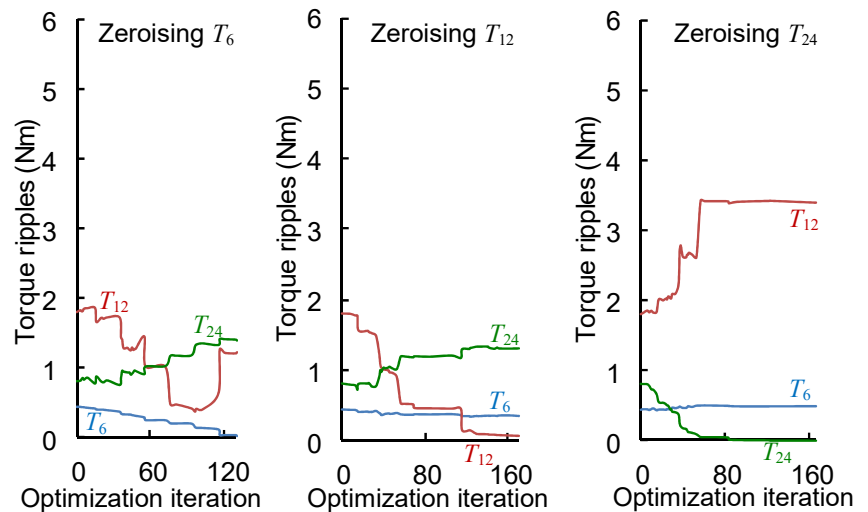

Fig. 17. Zeroization of specific order torque ripples under con. A.

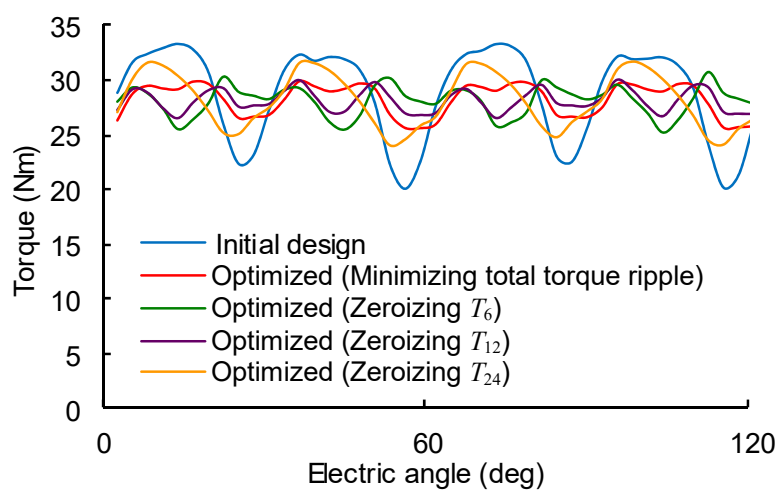

Fig. 18. Torque waveforms under driving condition A.

application. In this case, the final shape in the optimization of Fig. 16 is used as the initial design. The constraint conditions (a)-(d) are also imposed.

Fig. 17 shows the variation $T_{6}, T_{12}$, and $T_{24}$ with optimization iterations. Fig. 18 shows the torque waveforms of the initial and optimized motors. It is observed that each order torque ripple is reduced to be nearly zero when it is selected as the objective function. It implies that an arbitrary order torque ripple can be zeroized by optimizing the rotor surface shape under the specific driving condition due to the requirement of each application. On the other hand, Fig. 17 also indicates the tradeoff between the objective order torque ripple and the others. $T_{24}$ increases when $T_{6}$ or $T_{12}$ is selected as the objective function, whereas $T_{12}$ increases when $T_{24}$ is the objective function. However, these torque ripples are still significantly smaller than those of the initial design.

Fig. 19 shows the von Mises stress distribution of the optimized rotor and the decomposed torque ripple components under the condition A. In all the optimized motors, two dents are generated around the edges of the outside PM under the constraint condition of $\sigma_{\max }$. It is also confirmed that the phasors of $T_{6}, T_{12}$, and $T_{24}$ (red squares) are zeroized in each optimization from the initial value in Fig. 11 (right side, IPMSM, loaded), as follows:

a) $T_{6}$ is zeroized mainly by the variation in the phase angles of the components $B_{r, 5,-1} B_{\theta, 5,5}$ and $B_{r, 5,5} B_{\theta, 5,-1}$, which become nearly opposite to those of $B_{r, 7,1} B_{\theta, 7,7}$ and $B_{r, 7,7} B_{\theta, 7,1}$.

b) $T_{12}$ is zeroized mainly by the decrease in the amplitude of $B_{r, 13,1} B_{\theta, 13,13}$ and $B_{r, 13,13} B_{\theta, 13,1}$, while the increase in those of $B_{r, 11,11} B_{\theta, 11,-1}$ and $B_{r, 11,-1} B_{\theta, 11,11}$. The amplitudes of these components become nearly equal to each other, respectively.

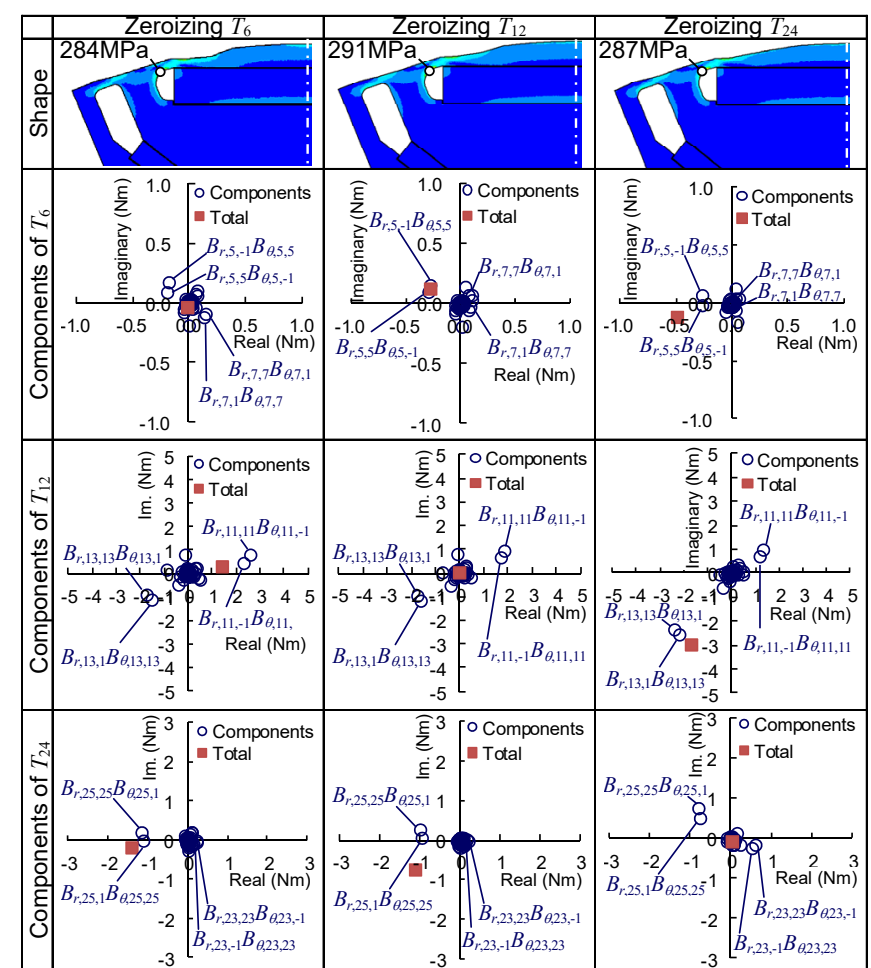

Fig. 19. Optimized rotor and torque ripple components under con. A.

c) $T_{24}$ is zeroized by both the variation in phase angles and amplitudes of the included components.

\section{CONCLUSION}

The mechanism of torque ripple generation by time and space harmonic magnetic field in PMSMs is investigated to obtain advanced motor designs. The harmonic fields that produce the torque ripples are specified by deriving the general expression between torque ripples and harmonic air-gap flux densities. The numerical and experimental verifications of the proposed torque ripple expression are carried out. Then, the difference of the torque ripple generation between SPMSMs and IPMSMs are discussed. From the observation by the proposed expression, the rotor surface optimization of an IPMSM is carried out to reduce the total and specific order torque ripples. As a result, following knowledges are obtained: a) $m$-th order torque ripple is generated by the product of the harmonic components of $B_{r}$ and $B_{\theta}$, whose space harmonic orders are identical to each other and the difference in the time harmonic orders is equal to $\pm m$.

b) There are considerable number of harmonic $B_{r}-B_{\theta}$ combinations that produce the torque ripple. The major components are the product of harmonic rotor MMFs and the harmonics generated by the modulation of fundamental field by stator standing waves.

c) The harmonic fields caused by rotor MMFs in SPMSMs are almost constant with load, whereas all the harmonic fields of IPMSMs vary with load because of the variation in rotor core saturation. As a result, the mechanism of the torque ripple generation in IPMSMs is more complex than SPMSMs

d) An arbitrary order torque ripple of IPMSMs under specific driving condition can be zeroized by optimizing the rotor surface shape without considerable deteriorations of the other 
important motor characteristics.

Further work will be required to consider the fluctuation of the manufacturing, which affects the torque ripples.

\section{REFERENCES}

[1] K. Yamazaki and H. Ishigami, "Rotor shape optimization of interior permanent magnet motors to reduce harmonic iron losses," IEEE Trans. on Industrial Electronics, vol. 57, no. 1, pp. 61-69, 2010.

[2] K. Yamazaki, Y. Kato, T. Ikemi, and S. Ohki, "Reduction of rotor losses in multilayer interior permanent-magnet synchronous motors by introducing novel topology of rotor flux barriers," IEEE Trans. Ind. Appl., vol. 50, no. 5, pp. 3185-3193, 2014.

[3] G. H. Lee, S. I. Kim, J. P. Hong, and J. H. Bahn, "Torque ripple reduction of interior permanent magnet synchronous motor using harmonic injected current," IEEE Trans. Magn., vol. 44, no. 6, pp. 1582-1585, 2008.

[4] F. Baneira, J. D. Gandoy, A.G. Yepes, and O. López, "DC-current injection with minimum torque ripple in interior permanent-magnet synchronous motors," IEEE Trans. Power Electronics, vol. 35, no. 2, pp. 1176-1181, 2020.

[5] M. Saur, D. E. G. Erazo, J. Zdravkovic, B. Lehner, D. Gerling, "Minimizing torque ripple of highly saturated salient pole synchronous machines by applying DB-DTFC," IEEE Trans. Ind. Appl., vol. 53, no. 4 pp. 3643-3651, 2017.

[6] K. Wang, Z. Q. Zhu, and G. Ombach, "Torque enhancement of surface-mounted permanent magnet machine using third-order harmonic," IEEE Trans. Magn., vol. 50 no. 3, 8100210, 2014.

[7] Z. S. Du and T. A. Lipo, "High torque density and low torque ripple shaped-magnet machines using sinusoidal plus third harmonic shaped magnets," IEEE Trans. Ind. Appl., vol. 55, no. 3, pp. 2601-2610, 2019.

[8] S. K. Lee, G. H. Kang, J. Hur, and B. W. Kim, "Stator and rotor shape designs of interior permanent magnet type brushless DC motor for reducing torque fluctuation," IEEE Trans. Magn., vol. 48, no. 11, pp. 4662-4665, 2012

[9] A. Rahideh and T. Korakianitis, "Analytical magnetic field distribution of slotless brushless PM motors. Part 2: Open-circuit field and torque calculations," IET Electr. Power Appl., vol. 6, no. 9, pp. 639-651, 2012.

[10] L. Li, K. M. Lee, K. Bai , X. Ouyang, and H Yang, "Inverse models and harmonics compensation for suppressing torque ripples of multiphase permanent magnet motor," IEEE Trans. on Industrial Electronics, vol. 65, no. 11 , pp. 8730-8739, 2018.

[11] Z. Q. Zhu and D. Howe, "Instantaneous magnetic field distribution in permanent magnet brushless de motors, Part IV: magnetic field on load," IEEE Trans. Magn., vol. 29, no. 1, pp. 152-158, 1993.

[12] H. Mikami. K. Ide, K. Arai, M. Takahashi, K. Kajiwara, "Dynamic harmonic field analysis of a cage type induction motor when magnetic slot wedges are applied," IEEE Trans. Energy Conversion, vol. 12, no. 4, pp. 337-343, 1997.

[13] K. Yamazaki and K. Utsunomiya, "Mechanism of Torque Ripple Generation by Time and Space Harmonic Magnetic Fields in Interior Permanent Magnet Synchronous Motors," International Conference on Electrical Machines (ICEM2020), Gothenburg, Sweden, pp. 1-6, 2020.

[14] A. Kameari, "Local force calculation in 3-D FEM with edge elements," Int. J. Appl. Electromagn. Mater., vol. 3, pp. 449-452, 1992.

[15] K. Yamazaki and Y. Kato, "Optimization of high-speed motors considering centrifugal force and core loss using combination of stress and electromagnetic field analyses," IEEE Trans. Magn., vol. 49, no. 5 , pp. 2181-2184, 2013.

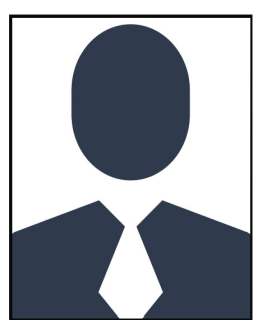

Katsumi Yamazaki (M'95) received the B.Eng. M.Eng., and Dr. Eng. degrees from Waseda University, Tokyo, Japan, in 1987, 1989, and 1996, respectively. In 1989, he joined Toshiba Corporation, Tokyo. Since 2007, he has been a Professor with the Chiba Institute of Technology, Narashino, Japan. His research interests include the analysis and the design optimization of motors. Dr. Yamazaki was the recipient of the Best Poster Paper Awards in the IEEE CEFC 2004, ICEM 2006, and IEMDC 2011; the Third Prize Paper Award of the Electric Machines Committee of the IEEE IAS in 2011; and the Best Paper Award in the IEEE Transactions on Energy Conversion in 2015.

Kento Usunomiya received the B.Eng. degree from the Chiba Institute of Technology, Narashino, Japan, in 2020, where he is currently working toward the M.Eng. degree. His main research interest focuses on the analysis of permanent magnet synchronous motors.

Hiroaki Ohiwa received the B.Eng degree from the Chiba Institute of Technology, Narashino, Japan, in 2020, where he is currently working toward the M.Eng. degree. His main research interest focuses on the analysis of permanent magnet synchronous motors. 